\title{
Infrastructured ad hoc networks
}

\author{
Anders Lindgren, Olov Schelén \\ Division of Computer Science and Networking \\ Department of Computer Science and Electrical Engineering \\ Luleå University of Technology, SE - 97187 Luleå, Sweden \\ E-mail: $\{$ dugdale, olov\}@sm. luth.se
}

\begin{abstract}
In traditional ad hoc networks, all nodes are considered equal and take equal part in the routing and forwarding of packets. We believe that this may not always be desirable. Rather, some nodes may be equipped with virtually unlimited power supplies, while others have to rely on battery power. Thus, we propose the creation of "infrastructured" ad hoc networks by the deployment of pseudo base-stations (PBSs), which are nodes that have constant power supply (e.g., through a power outlet or a car battery), do not move, and are present just to act as routers and forward packets for other nodes, thus allowing the mobile nodes to save power. The paper also presents ISAIAH, an ad hoc routing protocol based on the Ad-hoc On-demand Distance Vector $(A O D V)$ routing protocol, which tries to select routes that go through PBSs instead of through mobile nodes to reduce the amount of power spent by these mobile nodes. Furthermore, it allows nodes to enter a power-saving mode, significantly reducing the power consumption compared to AODV.
\end{abstract}

\section{Introduction}

Wireless networks are very popular due to their flexible nature, and the inherent possibility for wireless nodes to be mobile. Currently most wireless networks are infrastructured networks, where all communications go through a base station that acts as a gateway between the wired and wireless domains. To accommodate mobility, hand-over can be performed between two base stations as the wireless station moves from the coverage area of one base station to another, enabling the communication to seamlessly continue.

Infrastructure-less, or ad hoc networks have gained a lot of interest in the research community. In ad hoc networks there are no fixed routers or base stations, but instead all nodes have the capability to forward packets for each other. Because of the special properties of ad hoc networks such as quick topology changes due to mobility of the nodes, ordinary routing protocol fail to give good performance. There exist several ad hoc routing protocols [6] that enable ad hoc networks.

We do however see several problems with the traditional use of ad hoc networks and the scenarios where they are claimed to be useful. In this paper, we introduce the notion of infrastructured ad hoc networks that are intended for a different scenario than traditional ad hoc networks, namely as an extension of infrastructure wireless networks or to provide a temporary infrastructure at events where it is not desirable or possible to create an ordinary infrastructure. Such networks thus have different requirements than traditional networks. Therefore, we also present a protocol that can be used to realize such networks.

The rest of this paper is organized as follows. Sect. 2 gives an overview of the notion of infrastructured ad hoc networks and what is required of them, and Sect. 3 present ISAIAH, a modified version of the AODV ad hoc routing protocol, that supports infrastructured ad hoc networks. Sect. 4 describes the simulations run to evaluate the protocol, and the results of the simulations are shown in Sect. 5. Sect. 6 discusses some issues, and Sect. 7 concludes.

\section{Overview}

This section contains an overview of our thoughts on infrastructured ad hoc routing. Some of the "classical" scenarios where ad hoc networks have been claimed to be beneficial are at conferences, at disaster sites, and for military uses [2]. We do however claim that the use of "pure" ad hoc networks in such scenarios is somewhat limited. For example at a conference, it is most likely fairly easy to set up a base station, and further it is also very likely that the participants not only want to communicate with each other, but also with other hosts on the Internet. Another problem of ad hoc networks is the lack of incentive to forward packets for other users. A malicious user can use other nodes to forward its packets, while not wasting any of its own pre- 
cious battery power on forwarding packets for other nodes. In a system without malicious users, "power fairness" can become a problem since a node located close to a popular resource will forward lots of packets, thus draining its battery quickly.

We propose the introduction of a hybrid between the two worlds of infrastructure wireless networks and ad hoc networks. In such a network, ad hoc routing is used to extend the range of infrastructure wireless networks, or to create a temporary infrastructure that allows a larger area to be covered, while still not having to rely on mobile nodes for routing and packet forwarding. This can be done by deploying special nodes, "pseudo base stations" (PBSs), that have a high energy power source, low mobility, and take care of the majority of the routing of traffic in the network. These pseudo base-stations are basically only ordinary wireless nodes running an ad hoc routing protocol. The main difference is that since these have no demand on being mobile, they can e.g. be plugged into a power outlet and thus have a virtually unlimited power supply opposed to ordinary mobile nodes. Since power outlets are far more common than network connections, this could for example be used to provide complete coverage at a conference, a trade fair or a large sporting event. This would be accomplished by placing PBSs at strategic locations, provide them with "unlimited" power, and let them take care of forwarding packets towards their destination. By doing this, the mobile nodes won't have to participate in the exchange of routing messages and the forwarding of packets, thus reducing the power consumption of those nodes. Even if no power outlets can be found (e.g. at an outdoor event in the forest), power sources such as car batteries can provide the PBSs with power supplies that vastly exceed those of other nodes.

For such a network to be possible to create, special routing protocols are needed, that make use of the knowledge that some nodes are better than others to have in a route. We study requirements of routing protocols and mechanisms for realizing the "infrastructured ad hoc networks" described above. Our main contributions in this paper are the introduction of the concept of infrastructured ad hoc networks, and the design of a protocol suitable for such networks.

\section{ISAIAH}

This section describes Infra-Structure Aodv for Infrastructured Ad Hoc networks (ISAIAH), a modified version of the Ad hoc On-demand Distance Vector (AODV) routing protocol [5] that supports PBSs. The cost function used to calculate the cost of a route has been modified, there has been some changes in the way route requests are forwarded, and a power saving mode has been added. Sect. 3.1 gives a short introduction to the AODV routing protocol, Sect. 3.2 explains the new cost function, Sect. 3.3 and Sect. 3.4 explains the changes made to the forwarding of route requests, and Sect. 3.5 explains the power saving mode of ISAIAH.

\subsection{Introduction to AODV}

The Ad hoc On-demand Distance Vector routing protocol is, as the name suggests, an on-demand ad hoc routing protocol. When a node $\mathrm{S}$ needs a route to some destination $\mathrm{D}$, it broadcasts a route request $(R R E Q)$ message to its neighbors, including the last known sequence number for that destination. The RREQ is flooded through the network until it reaches a node that has a route to the destination. Each node that forwards the RREQ sets up a reverse route for itself back to node $S$. When the $R R E Q$ reaches a node with a route to $D$, that node generates a route reply that contains the number of hops necessary to reach $\mathrm{D}$ and the sequence number for D most recently seen by the node generating the reply, and unicasts that reply to S. All nodes that forward this reply back to the source $S$, create a forward route to D. The sequence number associated with the routes are used to prevent routing loops from occurring, and a route with a higher sequence number is always preferred over a route with lower sequence number. In order to maintain routes, AODV periodically transmits a HELLO message, with a default rate of once per second. Failure to receive three consecutive HELLO messages from a neighbor is taken as an indication that the link to the neighbor in question is down. If available, information from the link layer can be used instead of HELLO messages to detect link breakages, reducing the overhead. When a link goes down, any upstream node that has recently forwarded packets to a destination using that link is notified via a route reply with infinite metric for the destination. The node $S$ must then acquire a new route to the destination using the procedure above if it still wishes to communicate with that node.

\subsection{The cost function of ISAIAH}

In ISAIAH, we modify the "cost function" of AODV used to calculate the cost of a route, which should be minimized. The cost of a route in AODV is just the number of hops in the route, so the route with the shortest path is sought to find. ISAIAH on the other hand, has a more complex cost function shown in (1), where $C$ is the cost of the route, $h$ is the number of hops in the route, $H$ is the per hop cost, $M$ is the mobility cost, $m_{i}$ is the mobility of node $i$, $P$ is the power cost, and $p_{i}$ is the power scarcity of node $i$. This means that all "links" between nodes are not equally expensive to traverse, and the cost of a route is the sum of the costs of all the hops along that route. 


$$
C=\sum_{i=1}^{h}\left(H+M \cdot m_{i}+P \cdot p_{i}\right)
$$

Below, the different terms in the cost function are described in more detail. First there is a hop cost, $H$, that represent the cost of a hop. Assuming $H$ is set to 1 , this part of the cost will just be the hop count, which is sensible to include since if several routes are equal based on the other criteria, the shortest route should be selected. The "cost function" of AODV contains only this term.

We let each node have two additional properties, mobility $\left(m_{i}\right)$ and power scarcity $\left(p_{i}\right)$. The mobility property determines if a node is a PBS $\left(m_{i}=0\right)$ or a mobile node $\left(m_{i}=1\right)$. Power scarcity is how scarce, or valuable, power is to a certain node. Since one of the objectives of ISAIAH was to allow mobile nodes to save power from not participating in the routing, including power scarcity in the cost function has some benefits. If a route has to go through mobile nodes, it will choose a mobile node with much battery power (which can also increase the power fairness among nodes, so if some node has consumed much of its power on routing, another node is more likely to do the routing later on). Another example when this property can be useful is if someone plug their mobile unit to a power outlet. Then the power scarcity can be temporarily set to zero, so the node is more likely to help with packet forwarding (still, it is better to go through a PBS, since the mobile node might move away after a while, so the route through a PBS will be more stable). PBSs have a power scarcity of zero, and mobile nodes could for example let their power scarcity vary from 0.5 to 1 depending on the battery state. It is however likely that mobile nodes will just set $p_{i}$ to 1 to indicate that they consider power a scarce resource.

The mobility and power scarcity costs, $M$ and $P$, should be chosen to be larger than $H$ to ensure that the mobility and power scarcity of a route has greater impact on the choice of route than the hop count. As long as $M$ is sufficiently larger than $H$, routes through PBSs will be selected if they exist and are not significantly longer than the routes going through some mobile node. In the case where there exist no route going through only PBSs, the power scarcity will determine which mobile node will have to do the packet forwarding. Thus, the value of $P$ is less critical, but it should still be larger than $H$. We believe that in most cases it is sensible to give mobility and power scarcity approximately equal weight

\subsection{Changes in the forwarding of route requests}

To ensure correct operation the protocol, and to always select the "best" routes, a slight change had to be made in the way AODV forwards route requests. If AODV receives a route request with the same $\langle$ source, destination $\rangle$ pair, and the same sequence number as has been seen recently, it will just discard the route request packet. However, in ISAIAH this is not acceptable, since a route going through mobile nodes might be shorter (lower hop count) than the route with the lowest value of the cost function. Thus, using the method used in AODV would cause the protocol to settle with the first path (perhaps going through mobile nodes) on which a RREQ came to a node. Instead, in ISAIAH, the RREQ is checked, and if the cost of the new route is lower than the cost of the route stored in the table, the route request is forwarded (or replied to if this node has the route to the destination) even if the triple $\langle$ source, destination, sequence number $\rangle$ has been recently seen. Another problem with this approach is that we always have to do a network-wide search when trying to discover routes. In AODV, the RREQs start with a small TTL, thus only propagating in the vicinity of the source, and then increasing the TTL if no reply was received. In ISAIAH this can't be done if we are to ensure that the best route is to be found instead of just the shortest route. These changes causes some extra overhead, but the overhead will however be reduced by the proposition in the next section.

\subsection{The use of ISAIAH_TRIES to reduce overhead}

This section proposes a modification that will reduce the amount of routing packets traversing the network. This mechanism, called ISAIAH_TRIES, ensures that the first ISAIAH_TRIES times a mobile node sees a route request from a specific source to a destination (not counting multiple copies of the same RREQ message), it will discard it. Thus, only PBSs will forward it and participate in the route discovery. Each mobile node (i.e. not PBSs) should maintain two variables for each $\langle$ source, destination $\rangle$ pair, a counter $t_{\langle s r c, d s t\rangle}$ initialized to ISAIAH_TRIES, and $s e q_{\langle s r c, d s t\rangle}$, the last sequence number seen for that pair. Whenever a RREQ is received, the $\langle$ source, destination, sequence number $\rangle$ triple is inspected as shown in Algorithm 1.

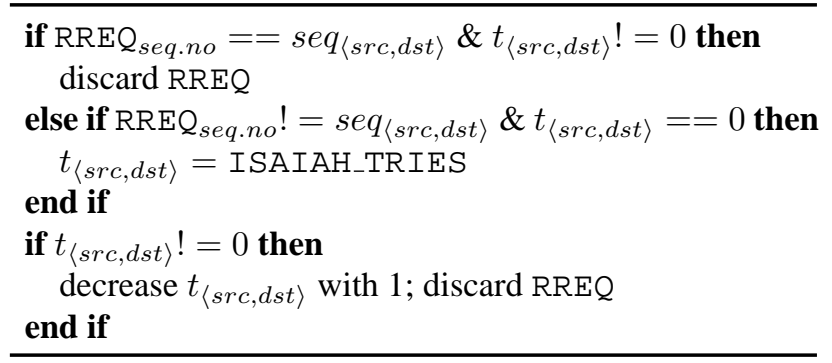

Algorithm 1: The ISAIAH_TRIES mechanism.

If the request didn't get discarded by this, it is processed as usual. This mechanism reduces the amount of routing packets sent. If no route to the destination can be found 
through only PBSs, the source will resend the route request, and this time the mobile nodes will also take part in the route discovery (if ISAIAH_TRIES is set to 1), and thus the route will be found eventually. This introduces additional delay in the route discovery process of routes that must contain mobile nodes, but as long as the coverage of PBSs is good, the impact of that should not be large.

\subsection{Power saving mode}

One major problem with designing power aware protocols is that it is not sufficient to just minimize the amount of data transmitted by each mobile node since the power consumption in idle mode is also rather high [1]. However, it is possible to put the network interfaces into "sleep" mode, where the power consumption is very low, but no data can be received or transmitted. It would be very beneficiary to let nodes enter this sleep mode for shorter periods of time, with the risk of losing data due to that being small. In this section we further extend ISAIAH, vastly improving the power saving capabilities of the protocol.

In addition to the normal HELLO messages used by AODV, we create two new messages, HELLO_PBS and HELLO_SLEEP. To enable the power-saving mechanism described below, information must be passed in these messages, so unlike in AODV we can no longer solely rely on information from the link layer, but the periodic transmission of HELLO messages is required. PBSs always utilize HELLO_PBS messages instead of HELLO message. Mobile nodes on the other hand can use both HELLO_SLEEP and HELLO messages. When a mobile node detects a HELLO_PBS message, it knows that at least one of its neighbors is a PBS, and thus assumes it will be OK to use power saving. At the next instant when the transmission of a HELLO message is scheduled, the node instead sends a HELLO_SLEEP message, indicating that this node is now entering the sleep mode. After that, the radio interface is powered down for a configurable period of time. This sleep time is also included in the HELLO_SLEEP message so surrounding nodes will know how long this node will sleep. A node will however always wake up in time to send the next HELLO message (the reception of such a message from a sleeping neighbor is also taken as an indication that the node is no longer sleeping). We recommend that at least one regular HELLO message is sent between two HELLO_SLEEP messages. In order to prevent the case where some neighbors already are asleep when a node $A$ notifies them that it is going to sleep (which means that they won't be aware that $A$ is asleep when they wake up), it is also possible for nodes to include the next time they plan to go to sleep in their normal HELLO messages. This means that nodes can predict when their neighbors will go to sleep next time even if they miss the sleep notification, thus reducing the risk of sending data to a sleeping node. If a HELLO message is indeed received, the information contained in that message of course supersedes the previously received information.

When a node receives a HELLO_SLEEP message it knows that the sender of that message won't be able to receive any data for a period of time. Thus, it suspends all packets it has in it's queue that has the sleeping node as their next hop. Packets arriving while the node is sleeping are also suspended. When the sleeping node wakes up again (detected either by the reception of a message from the node, or from a timer set to the sleep time expiring), the packets are activated again and can be sent.

\section{Simulations}

To find out how our changes affect the performance of the protocol, and to verify that ISAIAH indeed solves the problem of primarily using PBSs for routing and packet forwarding, we have implemented it in the $n s-2$ network simulator [4], and ran some simulations to evaluate the protocol. For comparison, we also did the same simulations using AODV. In the ISAIAH case, we have done the evaluation both with the power-saving mode described in Sect. 3.5, and without it (shown as "ISAIAH w/o PS" in the graphs in Sect. 5).

In our simulations we used a $1800 m \times 1000 m$ area where PBSs were placed as shown in Fig. 1. The range of the radio interfaces is $250 \mathrm{~m}$. The distances between adjacent PBSs were chosen to be slightly shorter than the range of the radios. In addition to the PBSs, 40 mobile nodes were also uniformly randomly placed in the area. These nodes move according to the "random waypoint" mobility model [3], i.e. they select a random destination and speed and move to that destination, pause there for a time, and then selects a new destination. The nodes moved at random speeds up to 5 $\mathrm{m} / \mathrm{s}$, and had a pause time of 15 seconds. Traffic was created using constant bit rate (CBR) UDP flows. A variable number of flows were set up between sources and destinations among the mobile nodes, starting at random points in time during the first 15 seconds of the simulation. For the cost function of ISAIAH we set $m_{i}$ and $p_{i}$ to 1 for mobile nodes, and 0 for PBSs, while $M$ and $P$ were set to 5 and $H$ set to 1 ; ISAIAH_TRIES was set to 1 . The simulations were run over 101 seconds of simulated time. In the cases of AODV and ISAIAH without power-saving mode, we did not use HELLO messages, but instead relied on link layer detection of neighbors since this lowers the overhead, and increases the performance of the protocols. When using ISAIAH with the power-saving mode, one regular HELLO message is sent between each HELLO_SLEEP message, and the interval between messages is one second.

Since power consumption is an important aspect of this work, it is important that the power consumption model of 
the simulator is accurate. Therefore, we extended the energy model in ns- 2 somewhat, loosely based on the real-life measurements done by Feeney and Nilsson [1].

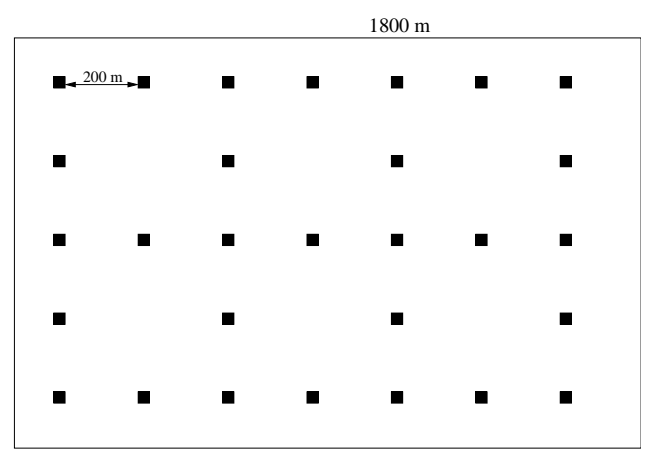

Figure 1. Scenario used for simulations. Placement of PBSs shown.

The metrics used for our evaluation are PBS forwarding percentage, routing overhead, normalized average path length, packet delivery ratio, and energy consumption. The PBS forwarding percentage is defined as $\frac{\# \text { packets forwarded by } P B S s}{\text { total \# forwarded packets }}$, and can be used to indicate whether or not a protocol actually manages to get most of the packets to be forwarded by PBSs. The routing overhead is the number of control packets sent and forwarded (measure network-wide). The normalized average path length shows how much longer the paths of ISAIAH are compared to the corresponding paths given by AODV. The packet delivery ratio is the fraction of data packets sent that are actually received at the destination. The energy consumption is measured as the average for all mobile nodes over the time of the simulation.

\subsection{Tuning of ISAIAH sleep time}

We ran some initial simulations to determine a good sleep time to be used for the power saving mode of ISAIAH in our performance evaluation. Fig. 2 shows the average power consumption of the mobile nodes for varying sleep times, and for two different traffic loads. As expected, the power consumption decreases as the sleep time increases. However, looking at Fig. 3, that shows the packet delivery ratio for the same sleep times, it is clear that a too long sleep time also affects the packet delivery ratio in a negative way. We therefore found a sleep time of 0.6 seconds to be a suitable tradeoff, and that is the sleep time that will be used in the simulations run to generate the results in Sect. 5 .

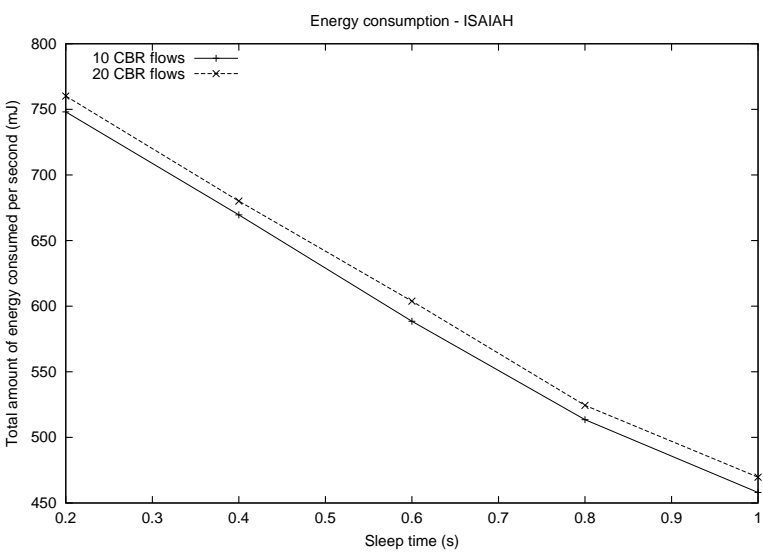

Figure 2. Energy consumption for varying sleep times of ISAIAH.

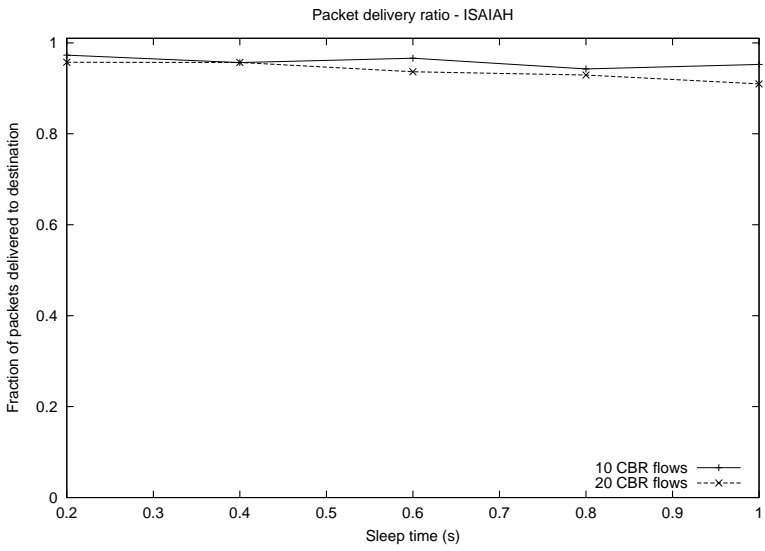

Figure 3. Packet delivery ratio for varying sleep times of ISAIAH.

\section{Results}

First of all we wanted to verify that ISAIAH is functional, and actually causes routes to go through PBSs. Fig. 4 shows how large fraction of all forwarded packets that are forwarded by PBSs. From this it is clear that ISAIAH actually selects routes through PBSs, since almost all packets are forwarded by PBSs. We can also see an indication of the fact that AODV treats the PBSs just like all the other nodes, since the fraction of packets going through PBSs is close to the fraction of all nodes that actually are PBSs.

The energy consumption of the protocols is shown in Fig. 5. The energy consumed by ISAIAH without powersaving is slightly less than for AODV, but they are still almost the same. Looking at the result from when using 


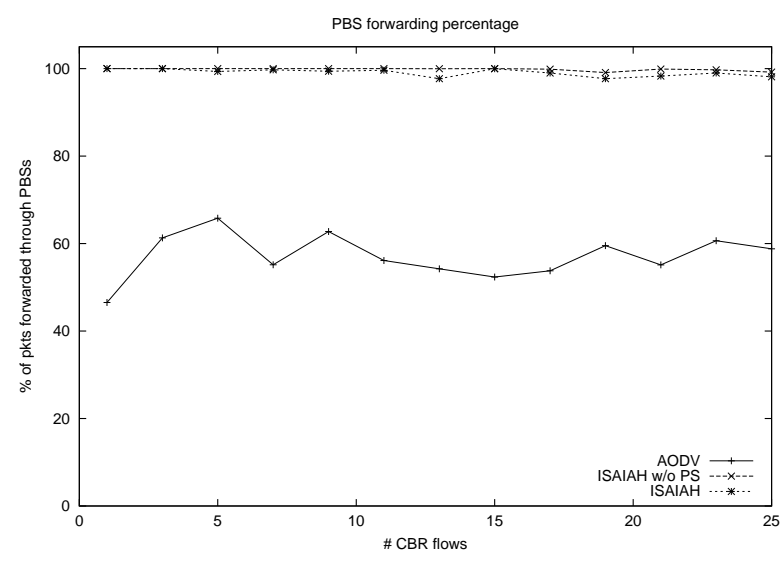

Figure 4. Fraction of packets forwarded that used PBSs.

ISAIAH with power-saving, it is clear that allowing nodes to enter the sleep mode is very beneficiary. The energy consumption of ISAIAH is almost $40 \%$ lower than for AODV.

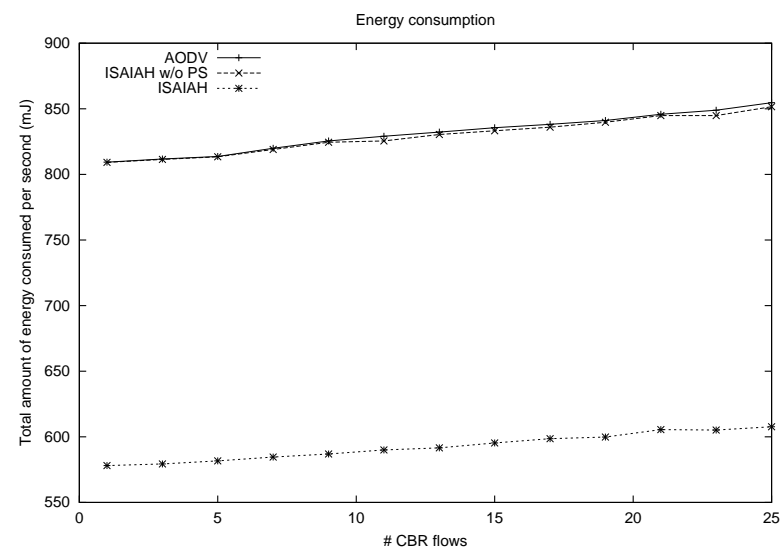

Figure 5. Energy consumption

In Fig. 6 we have studied the overhead imposed by the routing protocols by measuring the number of routing control packets transmitted on the wireless medium. We can see that ISAIAH has significantly lower routing overhead than AODV when no power-saving is used. The explanation to this is probably twofold. First of all, the use of the ISAIAH_TRIES mechanism heavily reduces the number of routing packets that has to be sent. Furthermore, since ISAIAH selects routes through the PBSs, there will be less broken routes that has to be repaired by new route discoveries. Since the power-saving mechanism requires HELLO messages to be periodically transmitted, the initial overhead of ISAIAH with power-saving is higher than for AODV. As the traffic load increases, the overhead for AODV does however grow quicker than for ISAIAH.

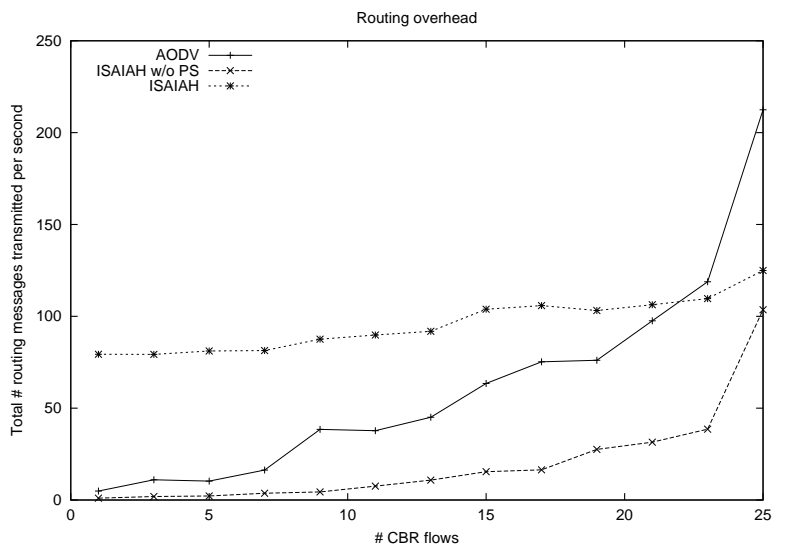

Figure 6. Routing overhead

Fig. 7 shows the fraction of sent packets that are actually received at the destination for the different numbers of CBR sources. ISAIAH without power-saving and AODV are able to deliver almost all packets (slightly higher delivery ratio for ISAIAH). When the power-saving mode is used. the delivery ratio is lower, but still acceptable.

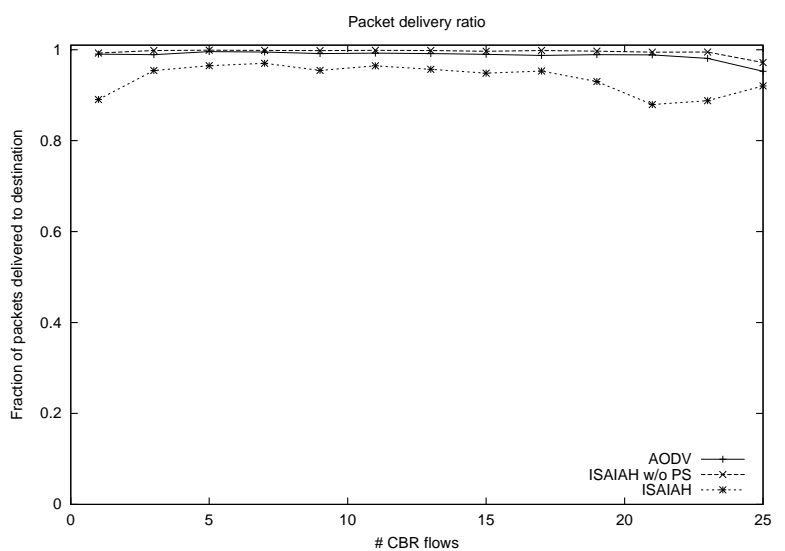

Figure 7. Packet delivery ratio for varying traffic load.

In Fig. 8 the average route length normalized with regard to AODV is shown. It can be seen that ISAIAH yield routes that are around $20 \%$ longer than the routes used with AODV for ISAIAH without power-saving, and somewhat longer when using the power-saving mode. This is rather natural since we only want to go through PBSs, and thus can't always choose the shortest path between the source and destination. However, the additional path length of ISAIAH is 
not very high, which is good since longer paths infer longer delays which affects performance negatively.

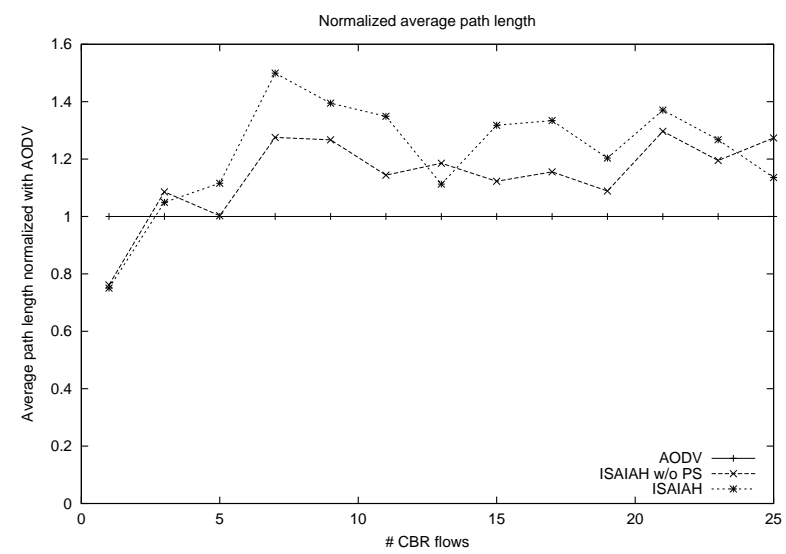

Figure 8. Average path length.

\section{Discussion}

Even though ISAIAH is working, and doing what it is supposed to do, there are still issues that need a more thorough investigation to develop an optimal protocol for infrastructured ad hoc networks. For example, it might be possible to make some smart decisions on when to enter the sleep mode, allowing nodes to spend more time sleeping.

An advantage of basing the protocol on the already existing AODV is that ISAIAH will be compatible with existing AODV implementation (although it will be unfair to AODV as AODV nodes will behave just like PBSs even if they are mobile). Furthermore, it also allows for simple implementation of ISAIAH by just making minor modifications to some existing AODV implementation.

Furthermore, the power-saving mode of ISAIAH could not only be used in conjunction with ISAIAH, but should also be possible to apply to AODV. In a scenario where there are no PBSs, and AODV is used, there is most likely much to be gained by being able to enter power-saving mode. The consequences of this should be further investigated.

\section{Conclusions}

In this paper we have presented the idea of infrastructured ad hoc networks, ad hoc networks where all nodes are not created equal. Instead, some nodes (which we call pseudo base-stations (PBSs)) are designed to take a greater responsibility for the routing. This reduces the amount of data regular mobile nodes need to transmit, thus also reducing the power consumption of those nodes.
We also present ISAIAH, a routing protocol for infrastructured ad hoc networks based on the AODV protocol. Our simulations show that the protocol is able to select routes that prefer PBSs over mobile nodes. The simulations also show that ISAIAH has lower routing overhead and packet loss than AODV in the scenarios used. Since the goal of ISAIAH is not to find routes with the lowest hop count, but routes through PBSs, routes might be longer than the corresponding route when using AODV. However, the simulations show that the average route length of ISAIAH is close to that of AODV. We also introduced a power-saving mode in ISAIAH, and when using that, the power consumption of the mobile nodes was significantly decreased.

\section{References}

[1] L. M. Feeney and M. Nilsson. Investigating the energy consumption of a wireless network interface in an ad hoc network environment. In Proceedings of IEEE Infocom, Anchorage, $A K$, April 2001.

[2] P. Johansson, T. Larsson, N. Hedman, B. Mielczarek, and M. Degermark. Scenario-based performance analysis of routing protocols for mobile ad-hoc networks. In Proceedings of Mobicom'99, Seattle, 1999.

[3] D. B. Johnson and D. A. Maltz. Dynamic source routing in ad hoc wireless networks. In Imielinski and Korth, editors, Mobile Computing, volume 353, chapter 5, pages 153-181. Kluwer Academic Publishers, 1996.

[4] S. McCanne and S. Floyd. ns network simulator version 2.1b8, June 2001.

[5] C. Perkins and E. Royer. Ad hoc on demand distance vector routing. In Proceedings of the 2nd IEEE Workshop on Mobile Computing Systems and Applications, pages 90-100, February 1999.

[6] E. M. Royer and C.-K. Toh. A review of current routing protocols for ad hoc mobile wireless networks. IEEE Personal Communications Magazine, 6(2):46-55, Apr. 1999. 